\title{
Cystic lymphangioma of the pancreas
}

\author{
IR Daltrey, CD Johnson
}

\begin{abstract}
Summary
Cystic lymphangioma of the pancreas is a rare condition. A 14-year-old girl presented with a cystic abdominal mass and abdominal pain. She was initially treated by biopsy and cyst drainage, and subsequently with partial excision of the cystic mass. The mass, which was a cystic lymphangioma involving the pancreas, recurred after each operation. Persisting symptoms led to a pylorus-preserving pancreatectomy and hepaticojejunostomy. Total excision is the only effective method of treating this benign tumour. The patient remains symptom free $2^{1 / 2}$ years after pancreatectomy.
\end{abstract}

Keywords: cystic lymphangioma, pancreas, abdominal mass

Intra-abdominal cystic lymphangiomas involving the mesentery, omentum, and retroperitoneum are rare benign tumours. Reported incidence rates vary from 1 in 25000 to 1 in 250000 hospital admissions. ${ }^{1}$ Cystic lymphangioma involving the pancreas is rarer still, only eight cases having been reported in the last 10 years. The majority of cystic lymphangiomas are discovered in childhood as asymptomatic abdominal masses.

\section{Case report}

A 14-year-old girl presented to a paediatric surgeon with an abdominal mass. She had complained of vague abdominal pains for the last year and on examination was found to have a swollen abdomen with a central abdominal mass. An ultrasound scan showed a large mixed solid and cystic mass, that extended from the left lobe of the liver to the splenorenal angle, lying anterior to the inferior vena cava and aorta. The pancreas could not be identified separately from the mass. The differential diagnosis suggested was a tumour of hepatic or ovarian origin.

Computed tomography (CT) showed that University Surgical Southampto General Hospital, IR Daltrey CD Johnson

Correspondence to Mr CD Johnson, University Surgical Unit, F Level, Centre Block, Southampton General Hospital, Tremona Road, Southampton SO9 4XY, UK

Accepted 13 November 1995
During follow-up over several months hề symptoms of abdominal discomfort and postprandial fullness returned. Repeated ultras nography confirmed continued enlargement of the mass and its origin from the pancreas. She was referred to us for further management.

At a third laparotomy in December $199 \overrightarrow{9}$ extensive cysts were found in the lesser sac and portahepatis encircling the duodenum and replacing the pancreatic head. Trial dissection to separate the mass from the pancreas faile $\Phi$ A pylorus-preserving pancreatectomy with hepaticojejunostomy was performed. Macro scopically, the specimen consisted of a multit cystic, multilocular mass, $14 \mathrm{~cm}$ in diametes diffusely expanding the body of the pancreas. Microscopy showed variably sized, massivefy distended, lymphatic channels with apparen origin within the pancreas (figure 2). $\mathrm{Th}$ diagnosis was of diffuse cystic lymphangion of the pancreas.

Two and a half years later she remains we with normal weight gain and developmenta with no evidence of further symptomatic cysto

\section{Discussion}

Wegner classified lymphangiomas by histolô gical appearance in $1877 . .^{1} \mathrm{He}$ separated the tumours into simple, cystic and cavernous lymphangiomas. ${ }^{2}$ Cystic lymphangiomas are classified as benign tumours but their exact origin is unknown. Many theories have beẹ, formed to account for their developmen including increased production or retention of lymphatic fluid, and infection or inflamma tion of the lymphatic channels. A developmental abnormality has also been blamed an this has led to cystic lymphangioma being classified as a hamartoma rather than a true neoplasm. ${ }^{1,2}$ Malignant transformations lymphosarcoma or adenocarcinoma have been documented ${ }^{3}$ but are exceedingly rare. ${ }^{4}$

Intra-abdominal cystic lymphangiomas may be multiple or single, unilocular or multw locular. ${ }^{2}$ It is believed that these differenat manifestations depend on whether there sequestration or obstruction of the lymphat vessels. If obstruction predominates then large unilocular solitary omental or mesenteric cys tend to develop. If budding or sequestration the lymphatic channels occurs then a typica cystic lymphangioma will develop that is multi locular and may be present in more than ore site. ${ }^{4}$ At operation it can sometimes be difficulf to differentiate mesenteric cysts from cystie lymphangioma. The correct diagnosis can obtained from histological examination. It important to obtain the correct histological 
Figure 1 (A) CT of the abdomen without intravenous contrast injection showing a large mixed solid and predominantly cystic mass (upper arrow) involving the pancreas (lower arrow). (B) The mass extends to the right side of the pelvis and distends the anterior abdominal wall

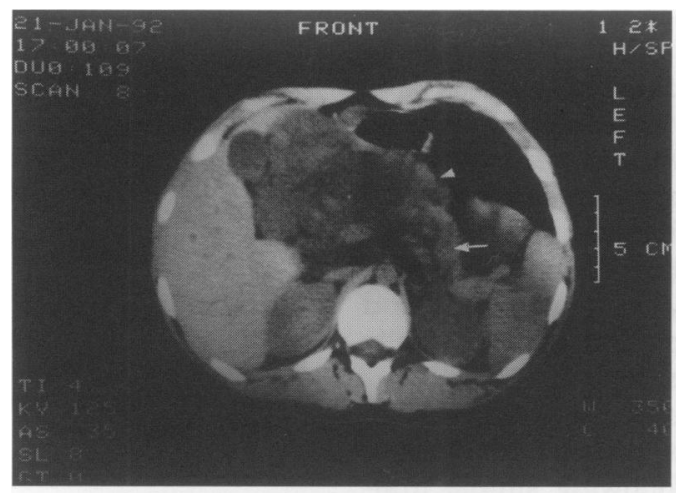

A

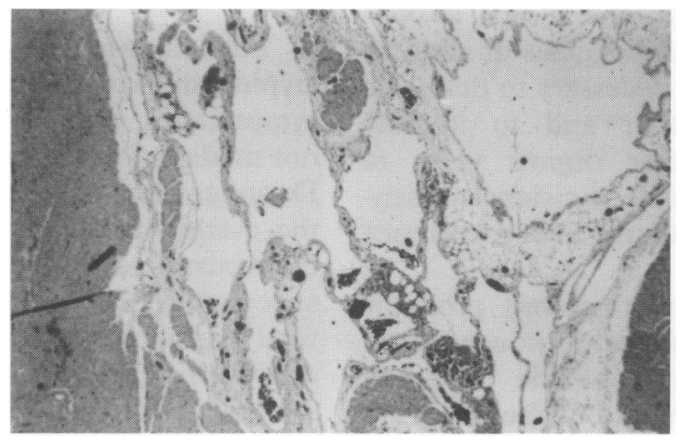

A

diagnosis because these two entities behave in completely different manners: cystic lymphangiomas are more aggressive and will invade other organs and hence the chances of complications or recurrence after excision are greater than for a mesenteric cyst. ${ }^{1,5}$

Cystic lymphangioma may occur at numerous sites throughout the body (box 1) but less than $1 \%$ are intra-abdominal. ${ }^{1,2,4,7}$ Retroperitoneal cystic lymphangiomas are usually a condition of childhood, $90 \%$ presenting before the age of two. ${ }^{2}$ The contents of the cysts can vary from serous to purulent, bloody to chylous. No difference in geographic distribution or sex predilection has been identified. ${ }^{1}$

The clinical presentation of these childhood tumours is varied. Small cystic lymphangiomas will remain asymptomatic and undetected. Larger growths may be detected incidentally on abdominal examination for an unrelated complaint. As growth of the mass occurs the patient may complain of abdominal distension and pain. Acute symptoms suggest pressure on neighbouring structures or peritonitis as a result of rupture, torsion, haemorrhage, or infection of the cyst. The commonest presentation is that of an asymptomatic abdominal mass. ${ }^{1,4}$

There is a wide differential diagnosis for children presenting with an abdominal mass. ${ }^{1,4,8}$ In the acute setting the commonest diagnosis made is that of acute appendicitis. ${ }^{1}$ Even with the aid of pre-operative investigation, the diagnosis of cystic lymphangioma is seldom made. Ultrasonography is the initial investigation of choice but, despite high sensitivity at detecting cystic lesions (94\%), the correct diagnosis will be suspected in less

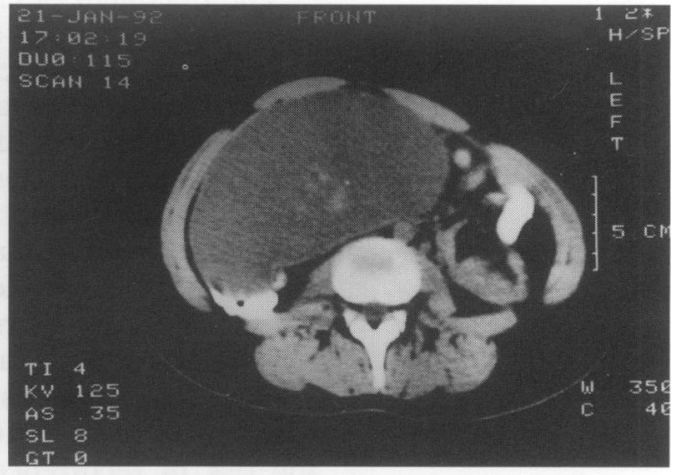

B

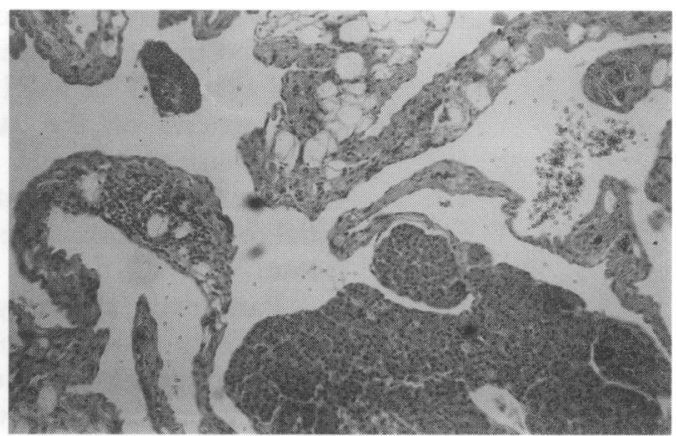

B

than $25 \%$ of patients. ${ }^{4}$ After CT and fine needle aspiration cytology the diagnosis is more likely to be considered. ${ }^{2}$ Magnetic resonance imaging (MRI) is equal to CT in demonstrating these cystic growths ${ }^{1}$ and avoids exposure to ionising radiation.

\begin{tabular}{|ll|}
\hline $\begin{array}{l}\text { Sites of distribution of cystic } \\
\text { lymphangiomas }\end{array}$ \\
\hline $75 \%$ & neck \\
$20 \%$ & axilla \\
$4 \%$ & mediastinum, lungs, chest wall, arms, back, \\
& $\begin{array}{l}\text { parotid, spleen, liver, pelvis, groin and } \\
\text { skin }\end{array}$ \\
$1 \%$ & retroperitoneum and omentum
\end{tabular}

Box 1

\begin{tabular}{|l|}
\hline Differential diagnosis of cystic \\
abdominal masses in children \\
\hline - ovarian cystadenoma or teratoma \\
- pancreatic cystadenoma \\
- renal: simple and multilocular cysts, polycystic \\
kidney disease, cystic nephroma, \\
hydronephrosis \\
- liver: choledochal cyst, cystic hepatoblastoma, \\
hydatid cyst \\
- splenic cyst \\
- intussusception \\
- enteric cyst or duplication \\
- mesenteric lipoma \\
- retroperitoneal neoplasm \\
\hline
\end{tabular}

Box 2 
The treatment of choice is total excision. If there has been invasion of other tissues then complete excision including the affected area of the involved organ is necessary. Partial resection is associated with a high recurrence rate, $50 \%$ in one series ${ }^{4}$ after a mean follow-up period of two years. After complete excision recurrences can occur but the rate is much lower at $7 \%$. Aspiration, cystenterostomy and peritoneal cavity marsupialization are usually followed by early recurrence.

Our case demonstrates the difficulty of diagnosing these lesions pre-operatively and the risk of recurrence following simple drainage or partial excision. Ultrasonography will confirm the cystic nature of the mass, however, the correct diagnosis will only be reached if intra-abdominal cystic lymphangioma is considered in the differential diagnosis of abdominal cysts. CT or MRI is necessary to delineate the extent of the tumour and to identify involvement of any other organs prior to planning surgery. Complete excision is essential to prevent an unnecessarily high recurrence rate.

Pancreatic cystic lymphangiomas probably arise from extension of a retroperitoneal cystic lymphangioma anteriorly into the pancreas. They represent the severe end of the spectrum

1 Roisman I, Manny J, Fields S, Shiloni E. Intra-abdomina lymphangioma. $B r \mathcal{F}$ Surg 1989; 76: 485-9.

2 Radin R, Weiner S, Koenigsberg M, Gold M, Bernstein R Retroperitoneal cystic lymphangioma. $A F R$ 1983; 140: 733-4.

3 Koshy A, Tandon RK, Kapur BM, Rao KV, Joshi K. Retroperitoneal lymphangioma: a case report with review of the literature. Am $\mathcal{f}$ Gastroenterol 1978; 69: 485-90.

4 Hebra A, Brown M, McGeehin K, Ross A. Mesenteric omental, and retroperitoneal cysts in children: a clinical study of 22 cases. South Med $\mathcal{F}$ 1993; 86: 173-6.

\section{Learning points}

- cystic lymphangioma of the pancreas is a rare, benign, tumour

- they usually present in infancy, $90 \%$ occuring before the age of two

- an asymptomatic abdominal mass is the commonest presentation

- acute symptoms are uncommon and tend to be misdiagnosed as appendicitis

- ultrasound combined with CT or MRI aids diagnosis and planning of surgery

- complete excision is essential to prevent recurrence

Box 3

of intra-abdominal cysts. Our patient was atypical in that she presented at age 14 with symptomatic mass. The correct diagnosis w not made before initial laparotomy and biopse. Drainage and subsequent partial excision of the cystic lymphangioma were soon followed by recurrence of the mass. She was treated successfully by partial pancreatectomy and total excision of the mass and she remains freet of symptomatic recurrence 30 months after surgery.

5 Takiff H, Calabria R, Yin L, Stabile BE. Mesenteric cysts an intra-abdominal cystic lymphangiomas. Arch Surg 198 120: $1266-9$.

6 Rossi L, Mandrioli R, Rossi A, Ugolotti U. Retroperitonea cystic lymphangioma. $B r f$ Radiol 1982; 55: 676-8.

7 Thomas AMK, Leung A, Lynn J. Abdominal cyst? lymphangiomatosis: report of a case and review of the lymphangiomatosis: report of a case

8 Sutton D. $A$ textbook of radiology and imaging. 3rd ed Churchill Livingstone, 1980. 